\title{
Lattice Boltzmann method used to simulate particle motion in a conduit
}

\author{
Jindřich Dolanský, Zdeněk Chára, Pavel Vlasák, Bohuš Kysela \\ Institute of Hydrodynamics of the Czech Academy of Sciences, v. v. i., Pod Pat'ankou 30/5, 16612 Prague 6, Czech Republic. \\ * Corresponding author. E-mail: dolansky@ih.cas.cz
}

\begin{abstract}
A three-dimensional numerical simulation of particle motion in a pipe with a rough bed is presented. The simulation based on the Lattice Boltzmann Method (LBM) employs the hybrid diffuse bounce-back approach to model moving boundaries. The bed of the pipe is formed by stationary spherical particles of the same size as the moving particles. Particle movements are induced by gravitational and hydrodynamic forces. To evaluate the hydrodynamic forces, the Momentum Exchange Algorithm is used. The LBM unified computational frame makes it possible to simulate both the particle motion and the fluid flow and to study mutual interactions of the carrier liquid flow and particles and the particle-bed and particle-particle collisions. The trajectories of simulated and experimental particles are compared. The Particle Tracking method is used to track particle motion. The correctness of the applied approach is assessed.
\end{abstract}

Keywords: Lattice Boltzmann method; Particle motion; Particle-fluid interaction; PIV; Particle tracking.

\section{INTRODUCTION}

The movement of solid particles in a liquid represents an important issue in recent hydrodynamic research, for example, suspended sediment transport or hydraulic conveyance of bulk material. The motion of particles generally consists of the interactions between the particles and the carrier liquid, mutual collisions of the particles, and their collisions with the conduit walls. The conveyed particles generally pass through different stages of movement such as sliding, rolling, saltation, or free suspension in the liquid flow (e.g., Abbott and Francis, 1977). To study the modes of particle motion some theoretical and numerical approaches employing Lagrangian models were suggested, for example, Wiberg and Smith (1985), Niño and Garcia (1994), and Lukerchenko et al. (2006, 2009). More recent advances in Lagrangian transport modelling are represented by Bialik et al. (2012). This deterministic approach can be combined with stochastic characteristics to pick up probability distributions of step lengths and resting times, for example, Bialik et al. (2015). To simulate bed load transport a microstructural approach can be used to model advection and dispersion of coarse particles carried by a turbulent stream (Ancey and Heyman, 2014). Experimentally-based models of bed load make it possible to identify unsteady phases of particle motion or describe distributions of particle velocities, accelerations, travel-times, for example, Campagnol et al. (2015) and Fathel et al. (2015).

Typically, the mathematical model of the particle-laden flows consists in formulation of the boundary problem by means of Navier-Stokes equations for the flow and solving the Newton equations for each particle, where the initial conditions of its motion are reassigned by every collision. The differential equations are then discretized by means of, for example, finite differences or finite volumes and subsequently simulated using a CFD (Computational Fluid Dynamics) method coupled with Discrete Element Methods (DEMs) for calculation of particle motion.

By contrast, the Lattice Boltzmann Method (LBM) represents a numerical strategy that makes it possible to simulate more complex particle-fluid systems and to solve them within a unique computational frame ( $\mathrm{Yu}$ and Fan, 2010). That means that both the action of the fluid on the particle and the action of the particle on the fluid can be expressed within the LBM based scheme. Thus the LBM simulation makes it possible to evaluate the mutual evolution of the particles and the flow and to calculate the instant response of the fluid on the particle.

The LBM was recently used as an alternative to traditional CFD methods for numerical simulations of a wide class of problems of fluid dynamics, for example multiphase systems (Ryu and Ko, 2012; Yan et al., 2011), dissipative particle dynamics, non-Newtonian fluid flows, suspension or biomaterials flows, and turbulence (Martinez et al., 1994). The LBM is a numerical approach, about three decades old, originating from the Lattice Gas Cellular Automata (LGCA) methods used for the simulation of complex fluid flows (e.g., Frisch et al., 1986; McNamara and Zanetti, 1988; Succi, 2001).

Specifically, the LBM is based on a microscopic model where the fluid is composed of interacting and propagating fictive particles along the Euler grid links. Interactions at the lattice nodes are defined by a collision operator that conserves mass and momentum. The collision and propagation steps comprise the lattice Boltzmann equation, which can be reduced to the macroscopic Navier-Stokes equations in the incompressible limit (Chen and Doolen, 1998) via the Chapman-Enskog expansion around the Knudsen number. The lattice Boltzmann equation can also be derived from the continuous Boltzmann equation by its discretization in space, time, and velocity space as well.

From the computational point of view, the most important advantage of the LBM based simulations, in comparison to the traditional CFD simulations, consists in the possibility of overall parallelization of the algorithm, which is enabled by its local dynamics. As the LBM ranks among explicit methods, it is quite straightforward to implement for both $2 \mathrm{D}$ and $3 \mathrm{D}$ cases. It is characterized by extraordinary flexibility in dealing with complex boundaries thanks to its particulate and local nature.

The 3D LBM based simulation is developed to study the motion of spherical particles in a closed horizontal conduit with a rough bed covered by stationary particles of the same size as the moving particles. Specifically, particle motion resulting from mutual interactions of the carrier liquid flow and the particles is evaluated with the help of the Momentum Exchange Algorithm (MEA), invented for this purpose by Ladd (1994) within the LBM approach and later modified by Aidun et al. (1998). To simulate sediment particle motion the LBM use to be modified and combined with different approaches which 
make it possible to improve interaction characteristics of moving particles and fluid flow, for instance, Liu et al. (2015) and Feng and Michaelides (2004).

Although the LBM based simulations may become unstable for high values of the Reynolds number, there are a variety of ways to prevent the instability. It is usually done either through modifications of the collision operator in the lattice Boltzmann equation - for example, by the Multi-Relaxation Time (MRT) method (e.g. Lallemand and Luo, 2003) or by the regularized LBM (Latt and Choppard, 2006) or by the so-called entropic LBM (Ansumali and Karlin, 2002; Karlin et al., 2006) - or through the selection of a suitable lattice boundary scheme, as it can substantially influence the solution. In the case of the examined process, the particle Reynolds numbers reach from $10^{3}$ up to $10^{4}$, and hence a specific lattice boundary scheme is used (Krithivasan et al., 2014). However, modifications of the LBM may also negatively influence either its efficiency or its accuracy.

To assess the accuracy, the simulated results are compared to the outputs of the experimental measurements. Specifically, the simulated trajectories are validated against the observed trajectories, which are gained by employing the PT (Particle Tracking) and PIV (Particle Image Velocimetry) methods. The presented results follow up tests and comparisons considered in the paper on 2D LBM simulation (Dolansky, 2014). Except for detailed qualitative analysis of compared trajectories, a quantitative comparison of heights and lengths is also performed.

The 3D simulation introduced in this paper yields the LBM based particle model enriched with the sophisticated boundary lattice scheme. Although it is surely not a new particle motion model conceptually, its realization and assessment are an important step to achieve such a model.

\section{EXPERIMENTAL METHOD}

Measurements were performed on an experimental pipe loop of smooth stainless steel pipes with an inner diameter $36 \mathrm{~mm}$ (see Figure 1a). A 2-m long transparent pipe viewing section (glass tube of inner diameter $40 \mathrm{~mm}$ equipped with a special optical box; see Figure 1b) was used for visual observation of the particle and carrier liquid flow patterns, which were recorded using a NanoSense MKIII+ digital camera. The particleliquid mixture was forced by a WARMAN 3/2 C - AH slurry pump from an open storage tank, and a variable speed drive was used to control the mixture flow rates. The mixture flow rate and concentration were measured by a KROHNECORIMASS- $800 \mathrm{G}+$ mass flow meter. The temperature of the mixture was maintained by the heat exchanger (Vlasák et al., 2012, 2014).

The moving particles are represented by glass balls of uniform size distribution, with a particle radius $r=3 \mathrm{~mm}$ and density $\rho_{p}=2540 \mathrm{~kg} \mathrm{~m}^{-3}$ (see Figure 2a). To measure the particle motion and velocity field in the fully stratified flow pattern, a stationary bed layer was created in the viewing section of the pipe. The stationary bed layer was created from two layers of spherical lead shots $\left(\rho_{b}=11340 \mathrm{~kg} \mathrm{~m}^{-3}\right.$, radius $\left.r=3 \mathrm{~mm}\right)$; its height from the pipe invert varied from 9 to $12 \mathrm{~mm}$, and the resulting bed roughness was $k=3 \mathrm{~mm}$ (see Figure 2b). Water was used as the carrier liquid.

The movement of glass balls in a pipe with a stationary bed is illustrated in Figure 2c. For lower flow velocities, the glass balls rolled along the pipe invert or saltated, mostly close to the pipe invert. With increasing flow velocity, the saltation became the dominant mode of particle movement, and the particles moving near the central part of the pipe reached higher velocities than particles moving in contact with the pipe invert

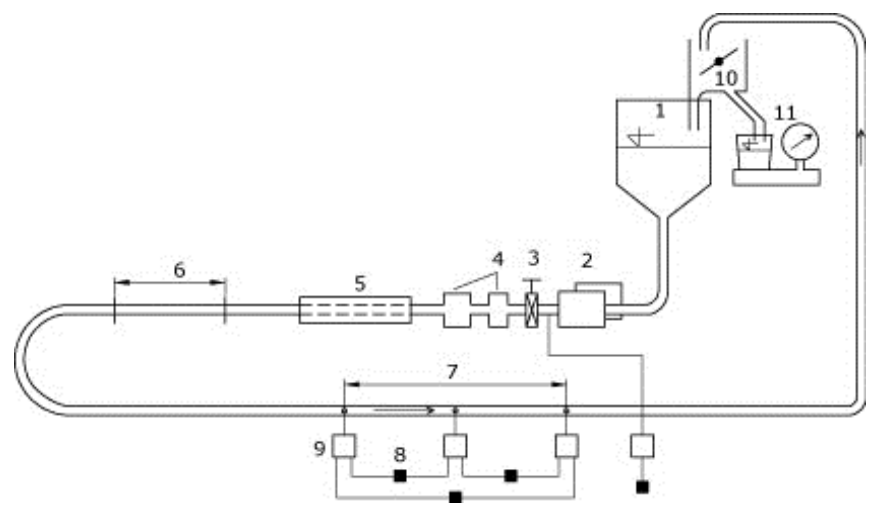

Fig. 1a. Schematic view of the experimental pipeline loop (1 slurry tank, 2 - pumps, 3 - control valve, 4 - flow meters, 5 - heat exchanger, 6 - transparent section, 7 - measurement section, 8 pressure transducer, 9 - sedimentation vessels, 10 - flow divider, 11 - density and discharge measurement).

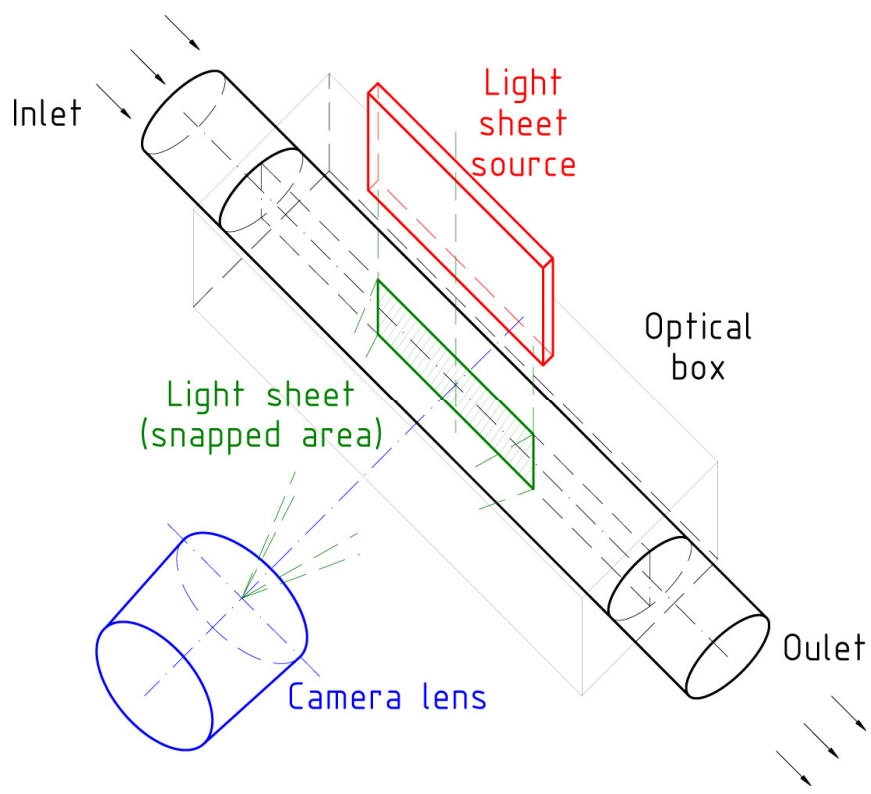

Fig. 1b. Schematic view of the optical measurement set-up in the transparent section.

(Vlasák et al., 2013) (see Figure 3).

The 2D PIV method was applied in the horizontal viewing section equipped with a special optical box around the glass pipe to evaluate the local water velocity $u$. A light sheet with a thickness of $1.5 \mathrm{~mm}$ was oriented in the streamwise direction on the centre line plane (see Figure 1b). Aluminium powder with a mean diameter of $10 \mu \mathrm{m}$ was used as the tracking particles of the flow. The images were recorded by a NanoSense MKIII+ high-speed camera with an image resolution of $1280 \times$ 512 pixels and frame rate of $1510 \mathrm{~Hz}$. The images were processed by the GPIV (http://gpiv.sourceforge.net) software package.

An interrogation area of $32 \times 32$ pixels was set up and the whole of the photographed area (Figure 1b) was evaluated. The evaluated velocity profiles were taken in the middle of the photographed area (evaluated vector field) to eliminate negative margin effects. Moreover, neither the values on boundaries at the pipe wall nor the disturbances caused by the vicinity of stationary particles on the bed were taken into account. The mean profiles (depicted in Figure 4) were obtained as averages from the whole record of each flow rate, which represented 3272 


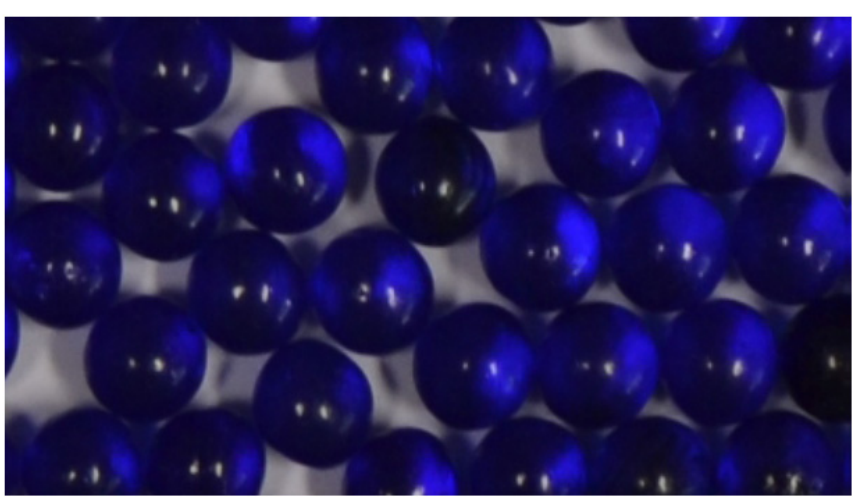

Fig. 2a. The moving particles are represented by glass balls $6 \mathrm{~mm}$ in diameter.

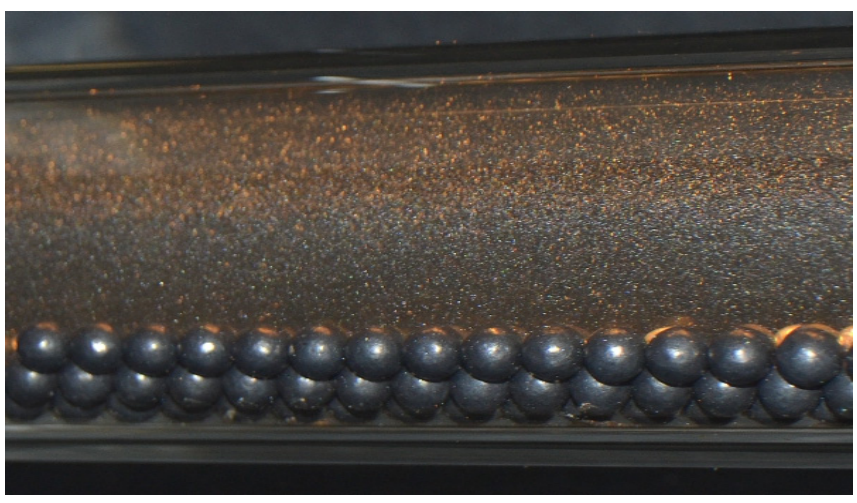

Fig. 2b. The stationary bed made from two layers of lead shots of the same shape and size as the moving particles. Scattered aluminium powder, which was used as fluid-flow tracking particles, can be seen above the bed.

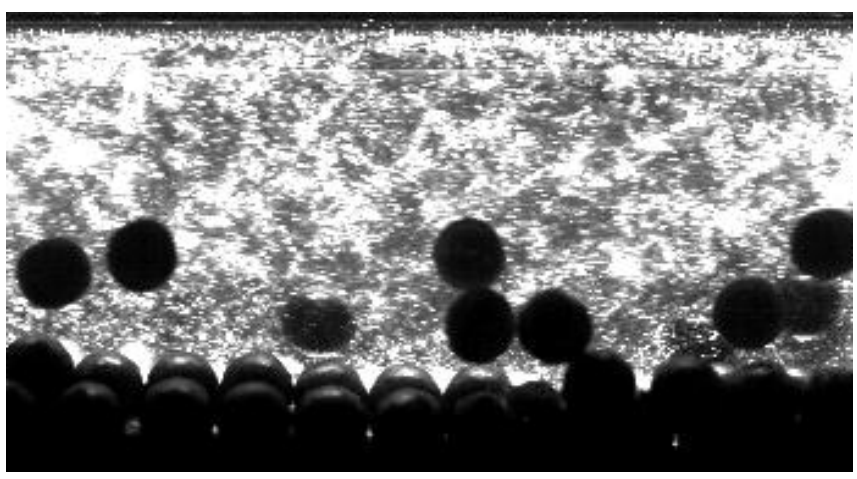

Fig. 2c. Glass balls conveyed by water in the pipe with the stationary bed.

frames (evaluated vector fields) with intervals of $0.662 \mathrm{~ms}$ (corresponding to a frequency of $1510 \mathrm{~Hz}$ ). The total record time was also determined and was approximately $2.17 \mathrm{~s}$.

The measured velocity profiles were asymmetrical due to the bed effect and the maximum velocity values were found at a distance of about $h=23 \mathrm{~mm}$ above the pipe invert. The velocity gradient was steeper in the lower part of the profile and local velocities near the stationary bed (from $h=9$ to $12 \mathrm{~mm}$ ) were practically equal to zero due to the high value of the bed roughness (see Figure 4).

The identical system of a high speed camera and light source used for the PIV measurements was also applied for the flow with moving glass particles. The motion of the glass balls were analyzed by a Particle Tracking (PT) method. The particle track-
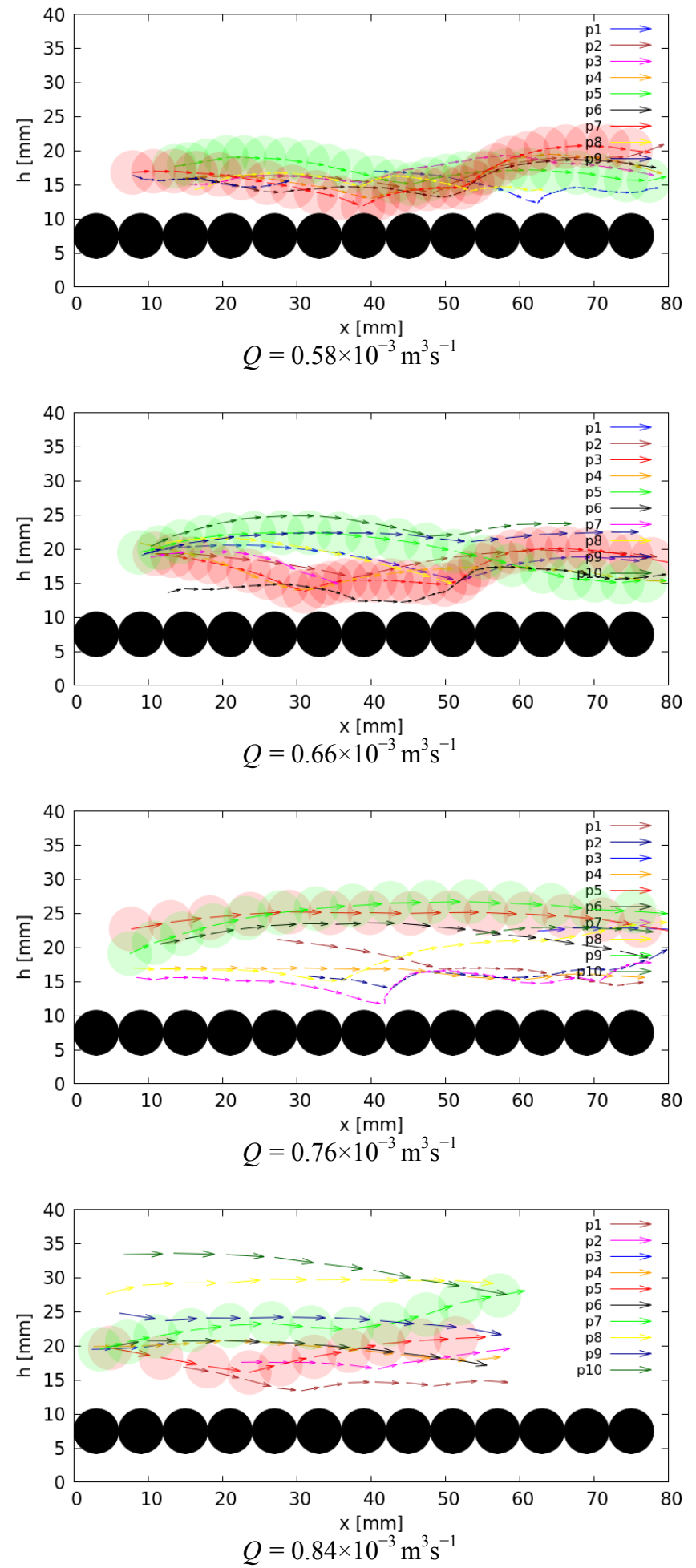

Fig. 3. Experimental particle trajectories in the pipe with the stationary bed. The simulated trajectories are highlighted by semitransparent circles.

ing was performed according to the idea of identification of the intensity of spherical particles (Martin et al., 1997). The evaluated region was selected and processed manually around the single tracked particle in each frame. The recorded image region was approximately $78 \times 42 \mathrm{~mm}$ with a resolution $1280 \times$ 680 pixels and a frame rate of $1560 \mathrm{~Hz}$. The image treatment was performed using the ImageJ package and the particle position was estimated with an accuracy higher than $0.2 \mathrm{~mm}$, that is, $0.06 r$. 


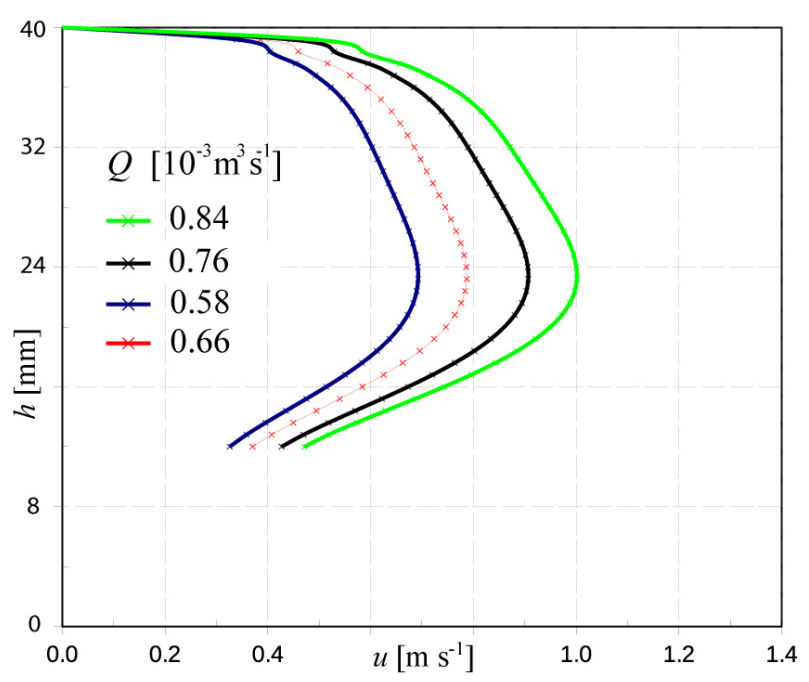

Fig. 4. The mean velocity profiles for different flow rates $Q$. The effect of the stationary bed made of spherical particles is manifested in the profile shapes.

\section{SIMULATION}

In the LBM, the fluid is composed of fictive particles - as opposed to macroscopic spherical particles moving in the flow - which interact in nodes and propagate along the lattice links with discrete velocities $\mathbf{c}_{\mathrm{i}}$. The in-house simulation employs the D3Q19 lattice (D stands for dimension and Q gives the number of discrete directions) to study the examined process.

\section{Basic algorithm}

The fictive particles are represented by particle distribution functions $f_{i}\left(\mathbf{x}, \mathbf{c}_{i}, t\right)$, which give the probabilities of finding a fictive particle in a node $\mathbf{x}$ with a certain discrete velocity $\mathbf{c}_{i}$ in time $t$. The collision and propagation process follows from the lattice Boltzmann equation:

$f_{i}\left(\mathbf{x}+\mathbf{c}_{i} \Delta t, t+\Delta t\right)-f_{i}(\mathbf{x}, t)=\frac{1}{\tau}\left(f_{i}^{e q}-f_{i}\right)$,

where the term on the right-hand side represents the frequently used Bhatnagar-Gross-Krook (Bhatnagar et al., 1954) collision operator, in which the change of the distribution function $f_{i}$ is proportional to its difference from equilibrium $f_{i}^{e q}(\Delta t$ is the lattice time step). The relaxation parameter $\tau$ then expresses the rate of relaxation to local equilibrium. The equilibrium distribution function $f_{i}^{e q}$ is given by the discretized Maxwell distribution of fictive particle velocities:

$$
f_{i}^{e q}=\rho w_{i}\left(1+\frac{\mathbf{c}_{i} \mathbf{u}}{c_{s}^{2}}+\frac{\left(\mathbf{c}_{i} \mathbf{u}\right)^{2}-c_{s}^{2} \mathbf{u}^{2}}{2 c_{s}^{4}}\right),
$$

where $w_{\mathrm{i}}$ are the weights normalized to unity, $\mathbf{u}$ is the velocity flow, and $c_{s}$ is the lattice sound speed. The collision operator fulfils the first law of thermodynamics, that is, conservation of mass and momentum. Macroscopic quantities of the flow, that is, fluid density $\rho$, momentum $\rho \mathbf{u}$, and momentum flux $\boldsymbol{\Pi}$, are obtained as moments over particle distributions $f_{\mathrm{i}}$ and discrete velocities $\mathbf{c}_{i}$ as

$\rho=\sum_{i} f_{i}, \mathbf{u}=\sum_{i} f_{i} \mathbf{c}_{i}, \boldsymbol{\Pi}=\sum_{i} f_{i} \mathbf{c}_{i} \mathbf{c}_{i}$.

\section{Boundary conditions}

To determine the influence of the (macroscopic) boundaries on the fluid, a lattice boundary scheme must be formulated for fictive particles. The macroscopic particles, walls, and bed have a solid boundary and the non-slip boundary condition is prescribed for them.

A number of lattice schemes are used to model this boundary condition depending on the shape of the boundary and requirements regarding accuracy and stability. As most of the object surfaces are not planar, their shape must be approximated by the stair-like surfaces, which bring additional instabilities and inaccuracies into the simulation.

The most well-known and frequently used lattice boundary scheme is the bounce-back algorithm, which ensures conservation of macroscopic mass and momentum. The bounce-back algorithm consists in inverting populations $f_{i}$ in boundary nodes:

$f_{i}=f_{-i}$

This scheme is considered to be of either the first order or the second order depending on the location of the boundary with respect to the closest fluid nodes and on the set of bounced-back populations in a boundary node.

The action of the particle on the flow is modelled with the help of the hybrid diffused bounce-back condition for the moving boundary, which combines some advantages of the standard bounce-back and of the diffusive boundary condition within the LBM (Krithivasan et al., 2014). Namely, it retains the locality of the bounce-back scheme and smooths populations $f_{i}$ in the immediate surroundings of the moving boundary. These conditions are realized in three steps: First the Ladd's two-sided bounce-back is applied according to Eq. (4) for boundary nodes in the fluid and in the solid, and then the modified fluid density $\bar{\rho}$ is computed at the boundary nodes and this density is finally used as an argument of equilibrium $f_{i}^{e q}$ at these nodes:

$$
f_{i}=f_{i}^{e q}(\bar{\rho}, \mathbf{v})
$$

where $\mathbf{v}$ is the velocity vector of the macroscopic particle. To refill populations in uncovered nodes, Grad's approximation is used:

$$
\begin{aligned}
& f_{i}^{\text {Grad }}= \\
& w_{i}\left(\rho+\rho \frac{\mathbf{c}_{i} \mathbf{u}}{c_{s}^{2}}+\frac{\sigma+\rho \mathbf{u}: \mathbf{u}-\rho \mathbf{u}^{2} \mathbf{I}-\left(p-\rho c_{s}^{2}\right) \mathbf{I}}{2 c_{s}^{4}}\left(\mathbf{c}_{i}: \mathbf{c}_{i}-c_{s}^{2} \mathbf{I}\right)\right),
\end{aligned}
$$

as it is more able to reduce fluctuations at these nodes ( $\boldsymbol{\sigma}$ is the stress tensor and $\mathbf{I}$ is the identity matrix).

The influence of the open boundaries, that is, inflow and outflow, on the solution is not negligible as it is known that the LBM approach suffers from pressure wave reflections caused by the compressibility effects of the method. The interaction between the open boundaries and the fluid domain is studied in, for example, Izquierdo et al. (2009).

The specified velocity profile at the inlet is implemented by evaluating equilibrium distributions for this profile. At the outflow boundary, the flow is supposed to leave the domain with minimal influence on the domain. The simple extrapolation scheme (Yu et al., 2005) 
$f_{i}\left(\mathbf{x}_{\text {out }}, t+\Delta t\right)=2 f_{i}\left(\mathbf{x}_{\text {out }}-\mathbf{c}_{i}, t+\Delta t\right)-f_{i}\left(\mathbf{x}_{\text {out }}-2 \mathbf{c}_{i}, t+\Delta t\right)$,

where $\mathbf{x}_{\text {out }}$ is the outflow boundary node and $\mathbf{c}_{i}$ is parallel to the streamwise coordinate, yields quite good results in comparison with other methods. On the contrary, the often used Zou-He boundary scheme (Zou and He, 1996), for example, which is based on inverting non-equilibrium part of distribution functions $f_{i}^{\text {neq }}=f_{i}-f_{i}^{e q}$, causes more significant disturbances in the domain.

\section{Particle motion}

The motion of a macroscopic particle is determined by means of the action of the fluid on the particle. The corresponding force of the flow is expressed by the difference $\Delta \mathbf{p}$ between the momentum of fictive particles, which income and leave the boundary of the macroscopic particle. The time rate of this momentum transfer $\Delta \mathbf{p} / \Delta t$ defines the hydrodynamic forces by which the flow acts on the objects:

$$
\mathbf{F}=-2 \sum_{\mathbf{x}_{b}} \sum_{i}\left(f_{i}-\frac{w_{i}}{c_{s}^{2}} \rho\left(\mathbf{c}_{i} \mathbf{v}\right)\right) \mathbf{c}_{i},
$$

which are calculated as a sum over momentum contributions from all fictive particles incident on the boundary nodes. The second term stands for the momentum contribution due to (macroscopic) particle motion. This approach is called the Momentum Exchange Algorithm and makes it possible to evaluate the force of the flow on the moving (macroscopic) object directly from the (fictive) particle distribution functions $f_{i}$. The additional contribution coming from the nodes uncovered by the particle motion is evaluated as equilibrium values $f_{i}^{e q}$ calculated from densities and velocities averaged over neighbouring fluid nodes.

Both the (macroscopic) particle-bed and particle-particle collision models are derived from impulse equations of the form

$m\left(\mathbf{v}-\mathbf{v}^{\prime}\right)=\mathbf{J}$,

which use the impulse force $\mathbf{J}$ as the measure of change of momentum (the quotation mark distinguishes the velocities before and after collisions). It is supposed that collisions take place in a very short time and all external forces can be neglected (e.g., Czernuszenko, 2009; Lukerchenko et al., 2009).

In the collision model, it was supposed that the friction coefficient of the glass particles corresponded to 0.8 and the restitution coefficient was approximately equal to one. Although the restitution coefficient achieves such values for high collision speeds, the elastic collision assumption was also accepted because the real values were not measured in the experiment.

To integrate Newton's equations of motion of the macroscopic particle, the leap-frog algorithm is chosen as it is simple, possesses second-order accuracy, and is invariant under time reversal (Allen and Tildesley, 1987).

\section{RESULTS AND DISCUSSION}

The results of the numerical simulation were compared to experimental outputs obtained with the presented experimental set-up. The simulated process took place in a cylindrical domain which corresponded to the optical box of the pipe where the particle trajectories were observed, see Figure 5. The cylin-

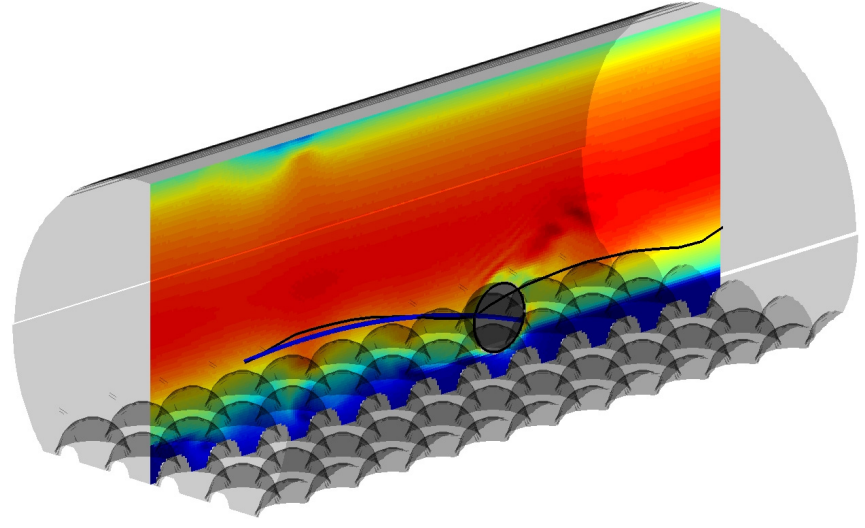

Fig. 5. Illustration of the simulated pipe domain and the location of the central plane onto which the trajectories are projected.

drical domain was inserted into the orthogonal domain with side determined by the pipe diameter. The resolution of the computational domain was given by the number of nodes per particle diameter. The requirement of 30 nodes over the particle diameter of $0.006 \mathrm{~m}$ resulted in a resolution of $400 \times 90 \times 200$ nodes, which corresponded to a physical domain of $0.08 \times 0.04$ $\times 0.04 \mathrm{~m}$. For this choice the lattice space step $\Delta \hat{x}=1$ corresponded to $\Delta x \sim 10^{-4} \mathrm{~m}$ in physical units. Although there is no straightforward way to choose $\Delta t$ with respect to $\Delta x$ it was assumed that the order of the time step and the space step are related as $\Delta t / \Delta x=0.1$, which is enabled by the hyperbolic nature of the lattice Boltzmann equation (e.g., Succi, 2001).

Trajectories of individual particles were evaluated for four different experimentally measured velocity profiles corresponding to flow rates $Q=0.58,0.66,0.76$, and $0.84 \times 10^{-3} \mathrm{~m}^{3} \mathrm{~s}^{-1}$ (see Figure 4). The measured velocity profiles were induced at the inlet of the simulated region to generate the flows within the domain. A couple of representative trajectories was chosen from a number of observed trajectories depicted in Figure 3 for each profile and compared to the path of the simulated particle. In the simulation, the selected particle was released from the positions $\mathbf{x}_{0}$ with velocities $\mathbf{v}_{0}$, which correspond to the experimental values observed at the initial instant of the measurement. Simulated trajectories are projected onto the vertical central plane (see Figure 5).

In Figure 6, eight images of the simulated and experimental trajectories on the background velocity fields can be seen for the four different velocity profiles. The couples in a row correspond to the same velocity profile or flow rate. Specifically, trajectories (a) and (b) correspond to the flow rate $Q=0.58 \times$ $10^{-3} \mathrm{~m}^{3} \mathrm{~s}^{-1}$, trajectories (c) and (d) correspond to $\mathrm{Q}=0.66 \times$ $10^{-3} \mathrm{~m}^{3} \mathrm{~s}^{-1}$, trajectories (e) and (f) correspond to $\mathrm{Q}=0.76 \times$ $10^{-3} \mathrm{~m}^{3} \mathrm{~s}^{-1}$, and trajectories $(\mathrm{g})$ and $(\mathrm{h})$ correspond to $\mathrm{Q}=0.84$ $\times 10^{-3} \mathrm{~m}^{3} \mathrm{~s}^{-1}$. The blue lines represent the simulated trajectories while the experimental trajectories are drawn in black lines. In the case of the collisionless trajectories $(e, f, h)$, the simulated trajectories reproduce the experimental ones very well except for the last one, that is (h), which undergoes additional particle-particle collision in the experiment. Trajectories with one collision with the bed, that is, $(\mathrm{d}, \mathrm{g})$, also correspond to the experimental ones quite well, although in case (g) the different collision location influences the resulting trajectory more significantly. The same is true for trajectories with two collisions with the bed, i.e. $(a, b, c)$. In the first case (a), the experimental particle obviously collides on the lower level of the bed - the bed in the experiment was not as perfectly regular as in the simulation - which causes a different subsequent evolution, while the simulated particle after the first collision undergoes 


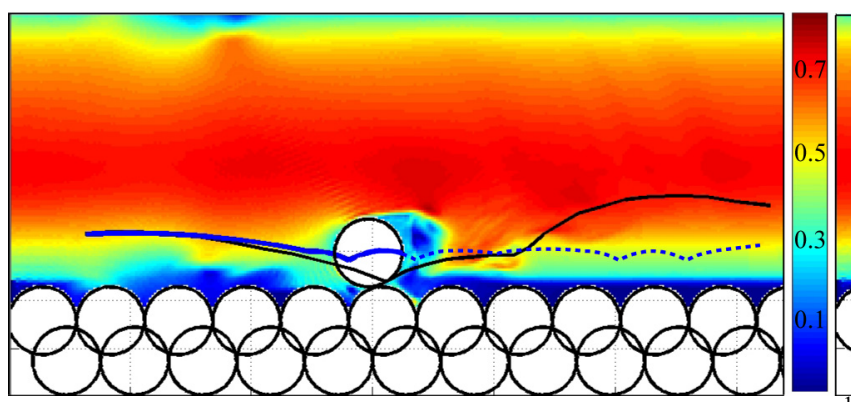

(a)

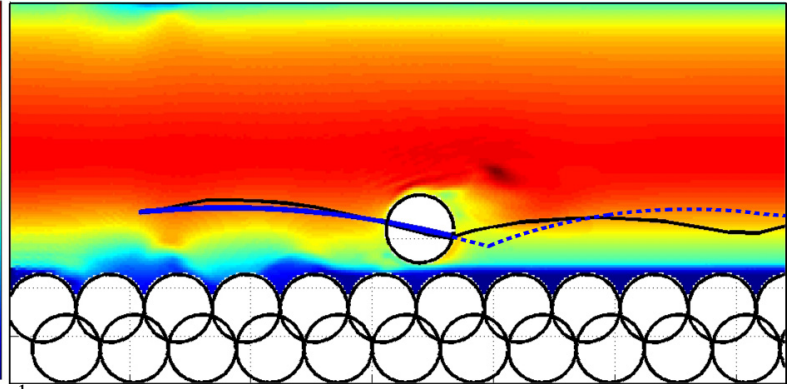

(b)

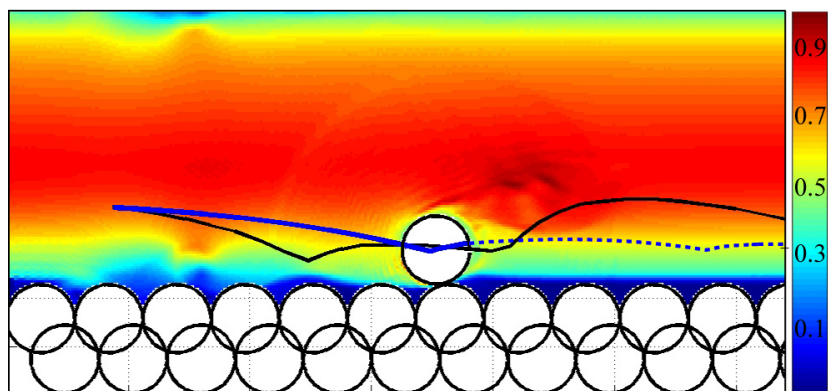

(c)

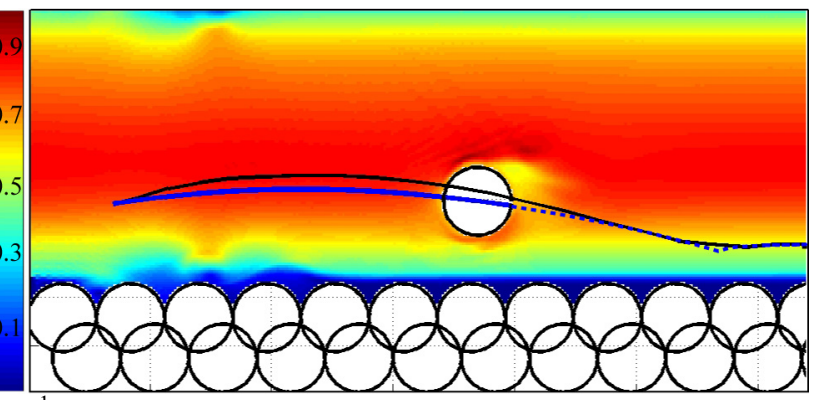

$u\left[\mathrm{~m} \mathrm{~s}^{-1}\right]$

(d)

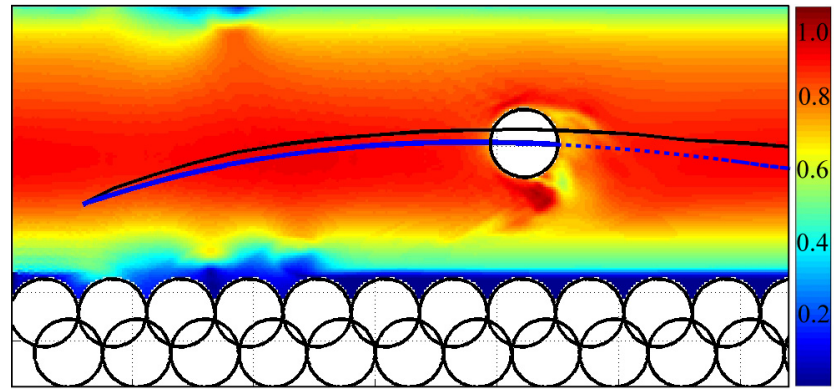

(e)

$u\left[\mathrm{~m} \mathrm{~s}^{-1}\right]$

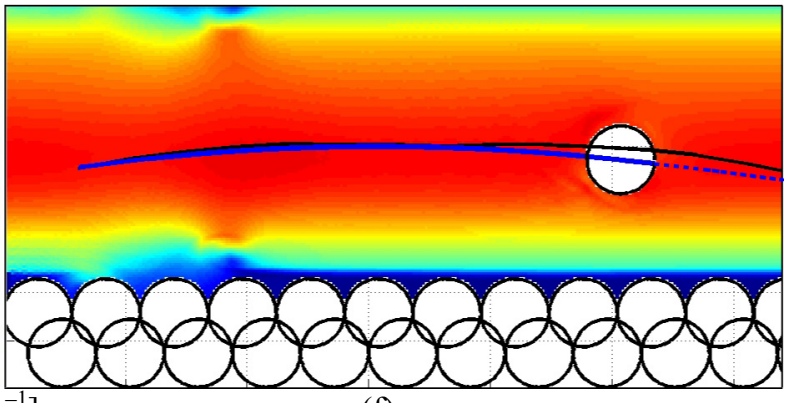

(f)

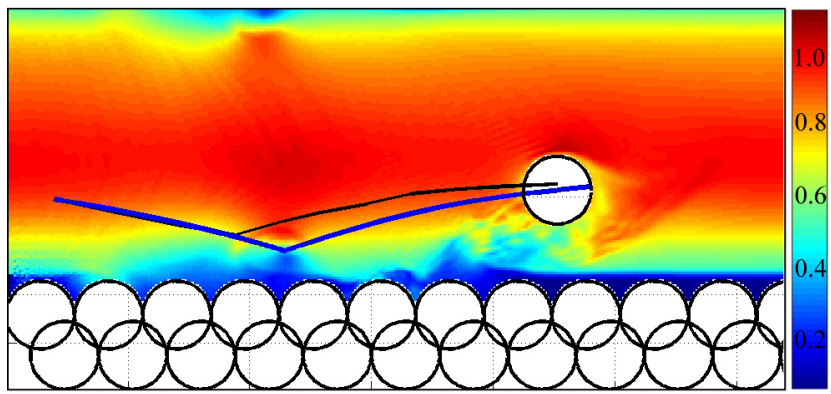

(g)

$u\left[\mathrm{~m} \mathrm{~s}^{-1}\right]$

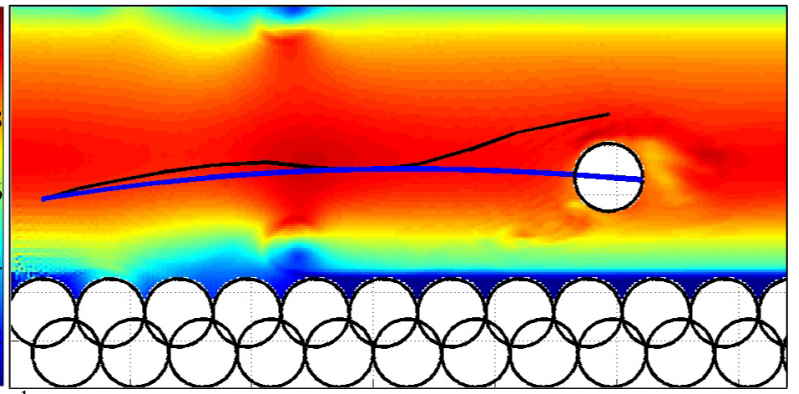

(h)

Fig. 6. Comparison of the experimental trajectories and simulated trajectories on the background of the velocity field. The blue lines represent the simulated trajectories while the experimental trajectories are drawn in black lines. Moving particles are displayed at the point where they arrived at the same time from the beginning of the simulation.

saltation motion above the bed. The simulated trajectory (b) follows the experimental one quite closely although it collides with the bed for the second time just outside the domain. Finally, the two-collision trajectory (c) differs most among all the compared trajectories, not only in the location of the first collision but also in the initial phase of the motion.

There are several factors that can cause differences between simulated and experimental trajectories. In the initial phase preceding any collision, the particles follow the experimental ones closely, except the trajectory (c). Differences can be ex- plained by gains or losses in velocity caused by the effects of insertion of the particles into the flow, which induces disturbances that affect the flow field and consequently the particle motion, or they can be attributed to the surrounding flow, which is influenced by the other particles.

After the collision with the bed, the shapes of trajectories are substantially influenced by the exact locations of the particlebed collisions as the bed is rough. Moreover, unexpected changes in the directions - as in ( $\mathrm{a}, \mathrm{c}, \mathrm{h})$ - can be caused by the interaction of an observed particle with another moving particle 
in the experiment. This behaviour is not reproduced by the simulated particle as it is assumed that the motion of particles consists only of free motion and collisions with the bed. Finally, differences between the simulation and the experiment can also be ascribed to the properties of the lattice boundary algorithms used as well as to the method itself with respect to the fact that the flow was turbulent and was characterized by a high Reynolds number.

From Figure 6, it is apparent that an unsteady flow arising around the moving particles influences the flow in the rest of domain only slightly, and consequently, they seem not to affect the velocity profiles significantly, which is also in accordance with the experimental results (e.g. Chara et al., 2016). However, with an increasing number of particles, the influence grows correspondingly. Figure 6 also illustrates the way in which vortex structures arise around the particles, especially during the contact with the bed. Notice also the wave-like disturbance, occurring in all parts of Figure 6, which results from interaction between waves arising after insertion of the particle into the flow and boundary conditions on the solid walls and at the inlet.

The aim of the simulation was to offer a tool that reproduces the behaviour of the particles and thus makes it possible to test theoretical models and assess the results of experimental measurements. In order to provide some quantitative measure of its correctness, four of the trajectories, namely $(b, d, f, h)$, were compared, specifically by the maximum height $h$ of the inner bounce of an experimental trajectory (see the locations of the maxima in Figure 7).

Moreover, as these trajectories are identical to the trajectories considered in Dolansky (2014), which were evaluated by a similar, but 2D, LBM based simulation, they could also be compared to them (see Figure 7 and Table 1). The 3D simulated and experimental trajectories are in blue and black respectively, as in Figure 6, while the 2D simulated trajectories are drawn in purple dashed lines.

From Figure 7 and Table 1 it is possible to deduce that until the particle collides with the bed, the results of both 2D and 3D simulations are similar, which can be interpreted as indicating that the particle does not significantly change its motion in the transversal direction (unless it collides with the bed).

In contrast, after the particle collides with the bed within the simulated domain, the $3 \mathrm{D}$ simulation gives obviously smaller maximum values due to the fact that in three dimensions the modulus of the particle momentum is decomposed into more components than in the 2D case, which corresponds more closely to the experiment. Thus it seems that particle motion is reproduced better in the $3 \mathrm{D}$ case as it agrees better in the phase after the collision.

It is important to realize that the simulated results are influenced by the fact that both particle motion and velocity profiles were experimentally measured in the central plane of the pipe, that is, in two dimensions, which had an impact on both the flow (induced by the $2 \mathrm{D}$ velocity profile at the inlet) and the particle motion, for which there was no reason to move transversally. This fact could explain the coincidence in the results of both 2D and 3D simulations in the initial phase of motion.

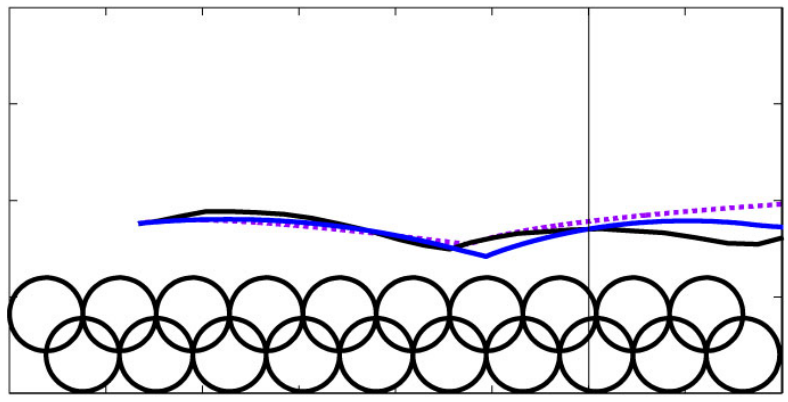

(b)

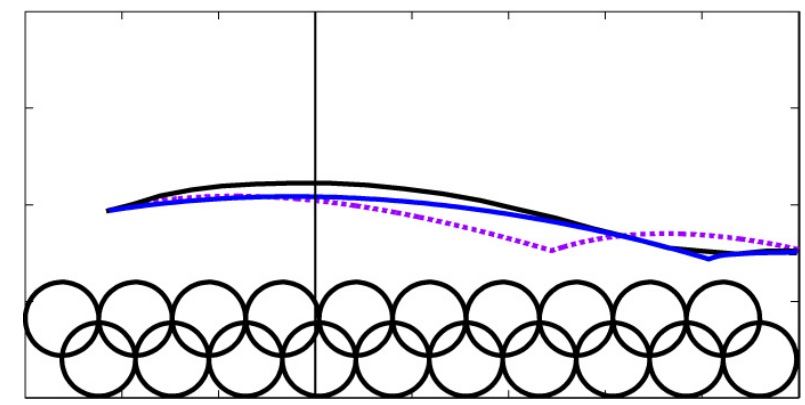

(d)

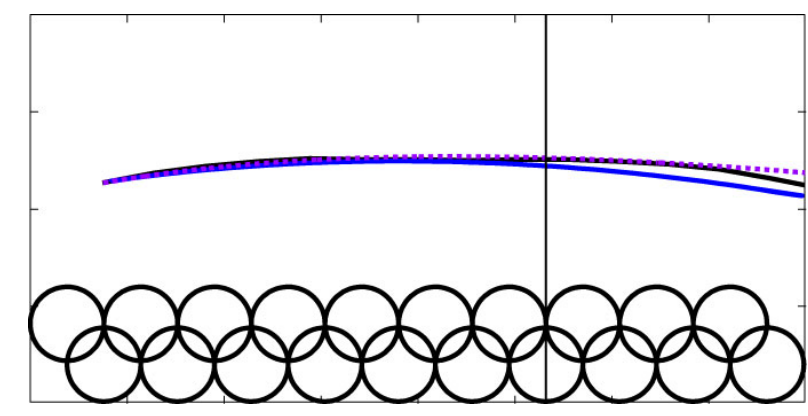

(f)

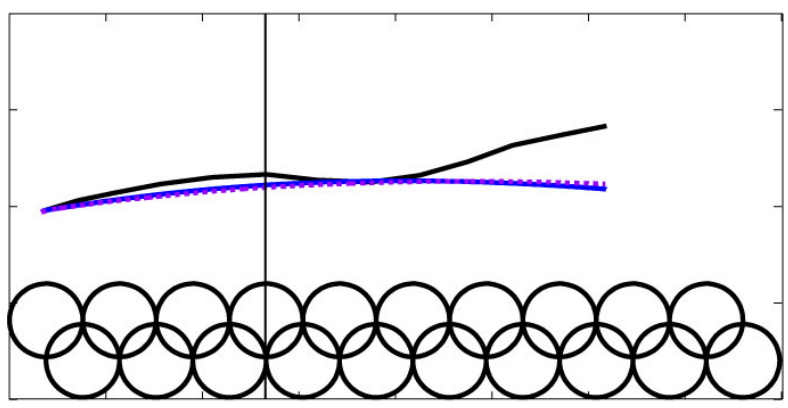

(h)

Fig. 7. Comparison of the experimental and $2 \mathrm{D}$ and $3 \mathrm{D}$ simulated trajectories. The 3D simulated and experimental trajectories are drawn in blue and black solid lines, respectively; the 2D simulated trajectories are drawn in purple dashed lines. The locations of the maximum height $h$ of the inner bounce are represented by the vertical lines.

Table 1. Comparison of the maximum heights $h$ of the bounce for four different flow rates.

\begin{tabular}{|l|c|c|c|c|}
\hline \multirow{2}{*}{ Trajectory } & Flow rate & \multicolumn{3}{|c|}{ Maximum heights $h\left[10^{-3} \mathrm{~m}\right]$} \\
\cline { 3 - 5 } & $Q\left[10^{-3} \mathrm{~m}^{3} \mathrm{~s}^{-1}\right]$ & Experimental & 2D Simulated & 3D Simulated \\
\hline $\mathrm{b}$ & 0.58 & 17.2 & 18.2 & 27.2 \\
\hline $\mathrm{d}$ & 0.66 & 22.5 & 20.7 & 2.9 \\
\hline $\mathrm{f}$ & 0.76 & 24.9 & 25.3 & 24.5 \\
\hline $\mathrm{h}$ & 0.84 & 23.1 & 22.2 & 2.4 \\
\hline
\end{tabular}




\section{CONCLUSIONS}

The process of particles being carried by a liquid in a closed pipe with a rough bed was studied. Both the particle motion and the fluid flow were simulated within the unified LBM based computational frame, which made it possible to study the mutual interactions of the carrier liquid flow and the particles and particle-bed collisions. The paper studied the moving boundary algorithm called the diffusive bounce-back condition, which is capable of balancing oscillations arising at the boundary. Particle movements induced by gravitational and hydrodynamic forces were evaluated with the help of the Momentum Exchange Algorithm. The trajectories of simulated and experimental particles were compared. The PIV measurements were used to measure the local velocity field to determine the velocity profiles and the PT was used to determine the experimental particle trajectories.

The simulation was - even for a rather coarse resolution stable due to the choice of the lattice boundary scheme, and computation was accelerated by using partial parallelization. Although the accuracy of the simulation was essentially influenced by the choice of the boundary conditions, there were further influences that followed either from the method itself or from the nature of the process (quite high Re).

As the process includes too many factors - flow, particles, bed, collisions, initialization, and so on - it is very hard to achieve a more accurate correspondence between the experiment and the simulation. It can be concluded that differences can be explained with respect to the conditions of both simulation and experiment and are qualitatively comparable.

Although the presented simulation surely does not represent a new particle motion model conceptually, its realization and assessment are an important step toward achieving such a model. For hydrodynamics applications, LBM based simulation represents a useful tool for studying particle motion in fluid flow, if accurate and correct. From the simulation results it is apparent that while the flow affects the movement of particles, the individual particle influences the flow less significantly, and this is also in accordance with the experimental results.

Acknowledgements. The support under project No. 15-18870S4 of the Grant Agency of the Czech Republic and RVO: 67985874 is gratefully acknowledged.

\section{REFERENCES}

Abbot, J.E., Francis, J.R.D., 1977. Saltation and suspension trajectories of solid grains in a water stream. Philos. Trans. R. Soc. London A, 284, 225-254.

Aidun C.K., Lu, Y., Ding, E.-J., 1998. Direct analysis of particulate suspensions with inertia using the discrete Boltzmann equation. J. Fluid Mech., 373, 287-311.

Allen, M.P., Tildesley, D.J., 1987. Computer Simulation of Liquids. Clarendon, Oxford.

Ancey, C., Heyman, J., 2014. A microstructural approach to bed load transport: mean behaviour and fluctuations of particle transport rates. J. Fluid Mech., 744, 129-168.

Ansumali, S., Karlin, I.V., 2002. Entropy function approach to the lattice Boltzmann method. J. Stat. Phys., 107, 291-308.

Bhatnagar, P.L., Gross, E.P., Krook, M., 1954. A model for collision processes in gases. I. Small amplitude processes in charged and neutral one-component systems. Phys. Rev., 94, 3, 511-525.

Bialik, R.J., Nikora, V.I., Karpiński, M., Rowiński, P.M., 2015. Diffusion of bedload particles in openchannels flows: distri- bution of travel times and second-order statistics of particle trajectories. Env. Fluid Mech., 15, 1281-1292.

Bialik, R.J., Nikora V., Rowiński, P.M., 2012. 3D Lagrangian modelling of saltating particles diffusion in turbulent water flow. Acta Geophys., 60, 6, 1639-1660.

Campagnol, J., Radice, A., Ballio, F., Nikora, V., 2015. Particle motion and diffusion at weak bed load: accounting for unsteadiness effects of entrainment and disentrainment. J. Hydr. Res., 53, 5, 633-648.

Chára, Z., Kysela, B., Dolanský, J., 2016. Saltation movement of large spherical particles. In: Proc. Int. Conf. of Numerical Analysis and Applied Mathematics 2016, Rodhes, Greece.

Chen, S., Doolen, G., 1998. Lattice Boltzmann method for fluid-flows. Ann. Rev. Fluid Mech., 30, 329-364.

Czernuszenko, W., 2009. Model of particle-particle interaction for saltating grains in water. Arch. Hydro-Eng. Env. Mech., $56,101-120$.

Dolanský, J., 2014. Simulation of particle motion in a closed conduit validated against experimental data. In: EFM14Experimental Fluid Mechanics 2014, Český Krumlov (CZ). EPJ Web of Conferences, vol. 92, pp. 115-120.

Fathel, S., Furbish, D., Schmeeckle, M., 2015. Experimental evidence of statistical ensemble behaviour in bed load sediment transport. J. Geophys. Res. Earth Surf., 120, 11, 22982317.

Feng, Z.-G., Michaelides, E.E., 2004. The immersed boundarylattice Boltzmann method for solving fluid-particles interaction problems. J. Comp. Phys., 195, 2, 602-628.

Frisch, U., Hasslacher, B., Pomeau, Y., 1986. Lattice-gas automata for the Navier-Stokes equation. Phys. Rev. Lett., 56, $1505-1508$.

Izquierdo, S., Martínez-Lera, P., Fueyo, N., 2009. Analysis of open boundary effects in unsteady lattice Boltzmann simulations. Comput. Math. Appl., 58, 914-921.

Ladd, A.J.C., 1994. Particles generally pass through different stages of motion such as rolling, saltation or suspension. J. Fluid Mech., 271, 311-339.

Lallemand, P., Luo, L.-S., 2003. Lattice Boltzmann method for moving boundaries. J. Comp. Phys., 184, 406-421.

Latt, J., Chopard, B., 2006. Lattice Boltzmann method with regularized pre-collision distribution functions. Math. Comput. Simul., 72, 165-168.

Liu, H., Ding, Y., Li, M., Lin P., Yu, M.H., Shu, A.P., 2015. A hybrid lattice Boltzmann method-Finite Difference Method model for sediment transport and riverbed deformation. Riv. Res. App., 31, 4, 447-456.

Lukerchenko, N., Chára, Z., Vlasák, P., 2006. 2D numerical model of particle-bed collision in fluid-particle flows over bed. J. Hydraul. Research, 44, 1, 70-78.

Lukerchenko, N., Piatsevich, S., Chára, Z., Vlasák, P., 2009. $3 \mathrm{D}$ numerical model of the spherical particle saltation in a channel with a rough fixed bed, J. Hydrol. Hydromech., 57, 2, 100-112.

Karlin, I.V., Ansumali, S., Chikatamarla, 2006. Elements of the lattice Boltzmann method I: Linear advection equation. Commun. Comput. Phys., 1, 616-655.

Krithivasan, S., Wahal, S., Ansumali, S., 2014. Diffused bounce-back condition and refill algorithm for the lattice Boltzmann method. Phys. Rev. E 89, 033313.

Martin, I.M.B., Marinescu, D.C., Lynch, R.E., Baker, T.S., 1997. Identification of spherical virus particles in digitized images of entire micrographs. J. Struct. Biol., 120, 146-157.

Martinez, D.O., Matthaeus, W.H., Chen, S., 1994. Comparison of spectral method and lattice Boltzmann simulations of two-dimensional hydrodynamics. Phys. Fluids, 6, 1285. 
McNamara, G.R., Zanetti, G., 1988. Use of the Boltzmann equation to simulate lattice-gas automata. Phys. Rev. Lett., 61, 2332-2335.

Niño, Y., García, M., 1994. Gravel saltation: 2. Modeling. Water Resources Res., 30, 6, 1915-1924.

Ryu, S., Ko, S., 2012. A comparative study of lattice Boltzmann and volume of fluid method for two dimensional multiphase flows. Nucl. Eng. Tech., 44, 6, 623-638.

Succi, S., 2001. The Lattice Boltzmann Equation for Fluid Dynamics and Beyond. Clarendon, Oxford.

Vlasák, P., Kysela, B., Chára, Z., 2012. Flow structure of coarse-grained slurry in a horizontal pipe. J. Hydrol. Hydromech., 60, 115-124.

Vlasák, P., Chára, Z., Kysela, B., Konfršt, J., 2013. Coarse grained particle flow in circular pipe. In: Proc. ASME Fluids Engineering Div. Summer Meeting, Incline Village, USA, vol. $1 \mathrm{C}$.

Vlasák, P., Kysela, B., Chára, Z., 2014. Fully stratified particleladen flow in horizontal circular pipe. Part. Sci. Tech., 32, 2, 179-185.

Yan, Y.Y., Zu, Y.Q., Dong, B., 2011. LBM, a useful tool for mesoscale modelling of single and multi-phase flow. Appl. Therm. Eng., 31, 649-655.

Wiberg, P.L., Smith, J.D., 1985. A theoretical model for saltating grains in water. J. Geophys. Res., 90, C4, 7341-7354.

Yu, Z., Fan, L.-S., 2010. Lattice Boltzmann method for simulating particle-fluid interactions. Particuology, 8, 539-543.

Yu, D., Mei, R., Shyy, W., 2005. Improved treatment of the open boundary in the method of lattice Boltzmann equation. Progr. Comput. Fluid Dyn., 5, 3-12.

Zou, Q., He, X., 1996. On pressure and velocity flow boundary conditions and bounceback for the lattice Boltzmann BGK model. Phys. Fluids, 9, 1591-1598.

\section{NOMENCLATURE}

\begin{tabular}{|c|c|c|}
\hline $\mathbf{c}_{i}$ & Discrete lattice velocity & $\left(\mathrm{L} \mathrm{T}^{-1}\right)$ \\
\hline$c_{s}$ & Lattice sound speed & $\left(\mathrm{L} \mathrm{T}^{-1}\right)$ \\
\hline$f_{i}$ & Fictive particle distribution functions $f_{i}$ & $(-)$ \\
\hline$f_{i}^{e q}$ & Equilibrium distribution function & $(-)$ \\
\hline$h$ & Height above the pipe invert & (L) \\
\hline I & Identity matrix & $(-)$ \\
\hline $\mathbf{J}$ & Impulse force on the particle & $\left(\mathrm{M} \mathrm{L} \mathrm{T}^{-2}\right)$ \\
\hline$k$ & Bed roughness & (L) \\
\hline$p$ & Fluid pressure & $\left(\mathrm{M} \mathrm{L}^{-1} \mathrm{~T}^{-2}\right)$ \\
\hline$Q$ & Flow rate & $\left(\mathrm{L}^{3} \mathrm{~T}^{-1}\right)$ \\
\hline$R$ & Particle radius & (L) \\
\hline$T$ & Time & $(\mathrm{T})$ \\
\hline $\mathbf{U}$ & Fluid velocity & $\left(\mathrm{L} \mathrm{T}^{-1}\right)$ \\
\hline $\mathbf{V}$ & Particle velocity vector & $\left(\mathrm{L} \mathrm{T}^{-1}\right)$ \\
\hline $\mathbf{v}_{0}$ & Initial particle velocity vector & $\left(\mathrm{L} \mathrm{T}^{-1}\right)$ \\
\hline$w_{\mathrm{i}}$ & Weights of discrete velocities & $(-)$ \\
\hline $\mathbf{x}_{0}$ & Initial position vector & (L) \\
\hline $\mathbf{x}$ & Position vector & (L) \\
\hline$\Pi$ & Fluid momentum flux & $\left(\mathrm{M} \mathrm{L}^{-1} \mathrm{~T}^{-2}\right)$ \\
\hline$\rho$ & Fluid density & $\left(\mathrm{M} \mathrm{L}^{-3}\right)$ \\
\hline$\rho_{p}$ & Bed particle density & $\left(\mathrm{M} \mathrm{L}^{-3}\right)$ \\
\hline$\rho_{p}$ & Particle density & $\left(\mathrm{M} \mathrm{L}^{-3}\right)$ \\
\hline $\boldsymbol{\sigma}$ & Fluid stress tensor & $\left(\mathrm{M} \mathrm{L}^{-1} \mathrm{~T}^{-2}\right)$ \\
\hline$\tau$ & Relaxation parameter & $(\mathrm{T})$ \\
\hline$\Delta \hat{t}$ & Lattice time step & $(\mathrm{T})$ \\
\hline$\Delta \hat{x}$ & Lattice space step & (L) \\
\hline$\Delta t$ & Physical time step & $(\mathrm{T})$ \\
\hline$\Delta x$ & Physical space step & (L) \\
\hline
\end{tabular}

Received 21 June 2016 Accepted 2 December 2016 